\title{
Apprivoiser les pères en protection de l'enfance
}

\author{
Annie Devault, \\ Professeure titulaire \\ Département de travail social \\ Université du Québec en Outaouais \\ C.P. 1250, Succ. Hull. \\ Gatineau, Québec, J8X 3X7 \\ Tél. : 819-595-3900 \#2506 \\ Courriel : annie.devault@uqo.ca
}

Chantal Zaouche Gaudron,

Chantal Zaouche Gaudron

Professeure de psychologie de

l'enfant UT2J, Maison de la

recherche Bureau 329, URM

LISST-Cers 5 allées A.

Machado 31058 Toulouse

Cedex 00(33)(0)6 82980290

Marie-Claude Huard-Fleury

Travailleuse sociale et assistante de recherche

Département de travail social

Université du Québec en Outaouais

\section{RÉSUMÉ}

Le présent article propose les résultats d'une recension d'écrits scientifiques dont l'objectif est de comprendre les différents facteurs qui interviennent dans le développement d'une relation de collaboration entre les pères et les professionnels dans le contexte de la protection de l'enfance. Les documents consultés présentent des résultats d'études scientifiques mesurant différents facteurs reliés à l'interaction entre les pères et les intervenants. L'analyse a permis de documenter le sujet sous différents angles : les croyances et préjugés des intervenants envers les pères, la formation professionnelle des intervenants et les facteurs associés au milieu de travail des services psychosociaux. Cette analyse fait également ressortir plusieurs obstacles à l'établissement d'une bonne relation père-intervenant de même que certaines dimensions qui favoriseraient une telle collaboration.

Mots clés: pères, intervenants, protection de l'enfance, collaboration, obstacles 
This literature review has the purpose of understanding the different dimensions that can be present in the development of a collaborative relationship between fathers and practitioners in the context of child protection services. This review is based on scientific documents. Most of the selected documents present research results on relationships between fathers and practitioners. The analysis of these documents allowed to look at this topic from different angles: beliefs and prejudices that practitioners may have towards fathers, practitioners' training and factors associated with their workplace. This analysis also reveals several obstacles in the development of a collaborative relationship between fathers and practitioners as well as some helping factors to build this kind of collaboration.

Key words: fathers, practitioners, child protection services, collaboration, obstacles

Les institutions de protection de l'enfance ont un statut particulier autant en France qu'au Québec, et en dehors des spécificités propres à chacun des deux pays, leur mandat, à caractère légal, vise à protéger les enfants des mauvais traitements qu'ils peuvent subir. Dans cette perspective, les intervenants oeuvrant au sein de cette institution incitent les parents à procéder à des changements sous peine de mesures aussi drastiques que le placement des enfants si les changements souhaités ne sont pas effectués.

Les relations entre les parents et les intervenants, dans ce contexte, sont importantes à examiner. Les parents doivent accepter la présence des services de protection alors qu'ils leur sont imposés suite à un signalement pour abus ou négligence. De leur côté, les intervenants, doivent développer une relation de collaboration avec les parents tout en remplissant leurs obligations vis-à-vis de la loi de protection des enfants. Comme l'indique Guay (2010) : «le défi clinique majeur sous-jacent au contexte légal, unique à ce milieu de pratique, est de réussir à concilier l'alliance thérapeutique avec le mandat de contrôle et de surveillance». (p.48).

Par ailleurs, les services de protection de l'enfance n'échappent pas à la tendance qu'ont généralement les institutions responsables du bien-être des enfants de s'adresser davantage aux mères qu'aux pères et de leur accorder une plus grande crédibilité en matière de soins des enfants. Ainsi, quoique l'on ait assisté depuis quelques décennies à des changements importants sur le plan des rôles parentaux, les institutions semblent encore peu s'ajuster à ces nouvelles réalités familiales.

La présente contribution propose les résultats d'une recension d'écrits scientifiques (articles de revues scientifiques, livres, ouvrages collectifs, documents résultant de groupes de travail gouvernementaux) issus principalement de recherches menées aux États-Unis, au Canada et particulièrement au Québec. Les documents analysés se basent sur des résultats de recherches ou des réflexions sur la paternité, la masculinité et leurs liens avec l'intervention psychosociale et, de façon plus spécifique, en contexte 
d'autorité. L'objectif de ce texte est de mieux comprendre les différents facteurs qui interviennent dans le développement d'une collaboration entre les pères et les intervenants dans les services psychosociaux, en mettant l'accent sur les obstacles et les facilitateurs du développement d'une telle relation en contexte de protection de l'enfance.

\section{Croyances et préjugés des intervenants quant aux rôles parentaux}

Les écrits scientifiques actuels sur les hommes évoluant dans les familles signalées aux services de protection de l'enfance se caractérisent par trois thèmes: l'invisibilité (Dominelli, Strega, Walmsley, Callahan et Brown, 2011), l'insignifiance (« unimportance ») et la dangerosité (Bellamy, 2009), témoignant ainsi de la réalité de la recherche qui serait davantage orientée vers une perspective déficitaire (déficits des pères) plutôt que constructive (leurs forces). Quelques recherches qui se sont penchées sur le point de vue d'intervenants au sujet des pères démontrent qu'ils sont, dans ce contexte également, relativement invisibles (Lacharité et Gagnier, 2015), perçus comme étant absents, menaçants et inutiles (Scourfield, 2003) ou que les intervenants se forgent une opinion des pères à partir des individus qui gravitent dans l'entourage du père (mère, grand-mère, intervenant) avant même de l'avoir rencontré (Maxwell, Scourfield, Featherstone, Holland et Tolman, 2012a). Des études indiquent aussi que les intervenants en contexte de protection entretiendraient une vision traditionnelle des rôles parentaux (Turcotte, 2014). L'attitude favorable envers les mères serait en partie attribuable au fait que les intervenants sont plus souvent en relation avec elles, et ils se fient à elles pour se forger une opinion des pères (Ewart-Boyle et al., 2013). Quant aux attributions négatives à l'égard des pères, elles prendraient ainsi leur source dans l'expérience des intervenants de contacts avec des pères considérés comme dangereux pour leurs enfants (Turcotte, 2014). En réaction à ces perceptions, des pères pourraient manquer de motivations à s'engager dans un processus d'intervention (Campbell, Howard, Rayford et Gordon, 2015).

Selon Zanoni et ses collègues (2013), les intervenants perçoivent que les attitudes et comportements des pères représentent un défi : ils réagissent plus fortement que les mères à certaines injonctions. Ces difficultés sont accentuées s'ils éprouvent des problèmes de consommation (alcool, stupéfiants...), s'ils présentent des conduites violentes ou s'ils font peu d'efforts pour contribuer au bien-être de leur enfant (Fletcher et St-Georges, 2010). Pour toutes ces raisons, les intervenants auraient tendance à adopter des attitudes méfiantes et parfois hostiles envers les hommes (O’Hagan, 1997). Les intervenants qui restreignent la présence des pères dans l'intervention peuvent, et parfois à juste titre, craindre pour la sécurité des enfants et de la mère, se méfier de l'agressivité possible du père et avoir peur de briser le lien de confiance avec la mère s'ils accordent trop de temps au père dans leurs interventions (Turcotte, 2014). 
Les expériences antérieures des intervenants avec des pères auraient toutefois une influence sur l'attitude qu'ils ont à leur égard. Ainsi, les intervenants qui ont vécu des expériences difficiles avec des pères, ont tendance à être moins enclins à faire des efforts pour les engager dans les pratiques d'intervention. Par contre, lorsque des intervenants ont vécu des expériences favorables avec les pères, ils s'intéressent davantage à qui ils sont, ils posent des questions pour mieux comprendre leur place et leur rôle dans la dynamique familiale et ont davantage tendance à croire qu'ils peuvent avoir des répercussions positives sur leurs interventions et dans la vie de leurs enfants (Campbell et al, 2015).

\section{Formation des intervenants}

La manière dont les intervenants sont formés influence aussi leur manière d'intervenir auprès des pères. Or, la théorie de l'attachement, qui accorde à la mère un rôle prépondérant dans le développement et le bien-être de l'enfant, est encore très présente dans la formation et dans la pratique des intervenants (Zanoni et al., 2013). De plus, encore aujourd'hui, les connaissances accumulées sur le développement des enfants se basent très largement sur des études portant sur les liens entre les enfants et les femmes de leur entourage (leur mère, leur éducatrice, leur enseignante) (Lacharité et Gagnier, 2015) et ce, malgré l'existence de plus en plus grande d'études confirmant l'impact positif de la présence des pères sur les enfants (Dubeau, Devault, Forget et Bizot, 2009). Il y a lieu de croire que la formation des intervenants repose sur des connaissances encore limitées du rôle du père. Il y a quelques années, au Québec, une équipe de recherche (deMontigny, Devault et Fleurant, 2009) s'est penchée sur la formation universitaire en travail social, en psychologie et en sciences infirmières, pour vérifier la présence de la thématique «paternité » dans les cours. Nous avons conclu qu'un très faible nombre d'enseignements aborde la question des pères dans la formation des intervenants. De plus, la majorité des professionnels ne sont pas formés pour travailler de manière spécifique avec des hommes notamment s'ils ont des problèmes de consommation, de violence ou de santé mentale. Ils se retrouvent aussi dépassés par la complexité des situations familiales que l'on retrouve en protection de l'enfance (Zanoni et al., 2013) et sont donc enclins à éviter les hommes et les pères, pensant ainsi simplifier les processus en jeu (Brown, Strega, Callahan, Dominelli et Walmslrey, 2009). Les résultats d'une recherche, auprès d'intervenants œuvrant dans les services de protection de l'enfance et dont l'objectif était d'examiner la perspective des intervenants du travail avec les pères, démontrent que ces intervenants ont besoin de formations sur les meilleures pratiques («best practices ») envers les pères, sur les modèles d'engagement paternel et sur les moyens de répondre aux besoins spécifiques des pères (Saleh, 2013). Une formation continue de courte durée qui explore les croyances et perceptions des intervenants et qui permet de démystifier certains préjugés semble donner des résultats probants pour sensibiliser les praticiens à l'intervention auprès des pères (Maxwell, Scourfield, Holland, Featherstone et Lee, 2012b). 


\section{Facteurs associés au milieu de travail des intervenants}

De façon générale, le travail dans le champ de la protection de l'enfance représente un défi particulier pour les intervenants qui œuvrent au quotidien avec des familles en grande détresse qui sont généralement réfractaires voire opposées à la présence de professionnels dans leur vie. Dans ce contexte, il est fondamental de tenir compte du climat émotionnel dans lequel l'intervenant évolue. Par exemple, la nécessité d'imposer des interventions dans une ambiance délétère peut engendrer une prise de distance émotionnelle. Cette distance due à une augmentation des situations et à leur complexité grandissante, aux politiques de performance, aux mesures de rendement, au stress engendré pour accompagner les vécus douloureux des familles serait pour eux une façon de se protéger, faisant place, parfois, à une déshumanisation de la pratique sociale (Howe, 2010).

L'étude d'O'Donnell et Waldo (2005) rapporte que les conditions de prestation des services ne tiennent pas compte des opinions et des expériences des pères. Les pères qui fréquentent ces services éprouvent ainsi des difficultés à se reconnaître dans les logiques institutionnelles puisqu'ils ne se sentent pas compris ou compétents comme parent, et adoptent en conséquence des attitudes, telles que la méfiance ou l'agressivité voire parfois la violence, qui peuvent compromettre leur lien avec les services et renforcer leur disqualification en tant que parent aux yeux des intervenants (Turcotte, 2014). Il est également décrit que les pères s'attendent à obtenir des réponses immédiates et à des indications claires, ce qui n'est pas toujours possible dans ce type de services (O'Donnell et Waldo, 2005). D'après la trentaine d'études recensées à propos des pères (Maxwell et al, 2012a), les intervenants notent qu'il est important de prendre le temps de bien comprendre les pères et leurs comportements plutôt que de les qualifier immédiatement de personnes agressives, les ignorer et ne plus les insérer dans les interventions proposées.

\section{Obstacles au développement des liens entre les intervenants et les pères}

Pour être bénéfique, le processus de soutien proposé par les services sociaux doit être associé à l'acceptation par les membres de la famille de leur vulnérabilité et à la reconnaissance de leur besoin d'aide. Ces dimensions se heurtent aux normes de la masculinité hégémonique (Welzer-Lang et Zaouche Gaudron, 2011) qui est plutôt construite autour de l'importance de maintenir son autonomie, de faire preuve de force et d'invulnérabilité (Devault, Huard-Fleury, Monette-Drevillon, Lacharité, deMontigny et Dubeau, 2015b). Même si on ne peut pas affirmer que tous les hommes suivent les dictats des stéréotypes associés à la masculinité hégémonique, il ressort d'une métasynthèse de recherches portant sur le rapport des hommes aux services (Roy, Tremblay, Guilmette, Bizot, Dupéré et Houle, 2014), que la quête d'autonomie figure au premier rang quand il s'agit de comprendre le rapport des hommes avec les personnes de leur entourage et avec les problèmes qu'ils éprouvent. Cette 
réalité est présente autant chez les hommes plus jeunes que chez les hommes matures. La socialisation masculine induirait donc un obstacle à se montrer vulnérable, à exprimer ses émotions et à demander de l'aide (Roy et al, 2014). Or, dans le contexte des services psychosociaux, et en particulier en contexte d'autorité, cette notion d'autonomie est clairement mise à mal. On attend des parents qu'ils reconnaissent leur besoin d'aide et donc qu'ils affichent une certaine vulnérabilité et avouent avoir besoin d'aide. Chez les hommes, et encore davantage chez les hommes qui adoptent les comportements et attitudes associés à la masculinité hégémonique, ces difficultés à exprimer leurs besoins d'aide seraient bien présentes (Zaouche-Gaudron, Jayr et Kettani, 2005). Les démarches des intervenants peuvent alors se heurter à de vives réactions de la part des pères qui se trouvent «vulnérabilisés » par le discours des intervenants.

Selon Ewart-Boyle et ses collègues (2013), un intervenant qui miserait d'abord et avant tout sur l'expression des émotions vécues dans la situation problématique, serait confronté à davantage de difficultés s'il est face à un homme qu'à une femme. De même, un mode bureaucratique et impersonnel appellerait des réactions hostiles et de résistance de la part des parents, ce qui diminuerait les chances de créer une relation constructive, de collaboration (Howe, 2010). De plus, une communication basée sur la confrontation, un faible niveau d'écoute et d'empathie (Forrester et Harwin, 2008) ou un mode d'intervention procédural, informationnel, qui ne favorise pas la participation du parent ne serait pas un gage de succès (Maxwell et al., 2012b). Les pères interrogés dans l'étude de Devault, Forget et Dubeau (2015a) ont confirmé que l'obligation d'interventions et les directives rigides les amènent à les rejeter. Il semble toutefois que les intervenants qui perçoivent que les réactions hostiles des pères ne sont que le reflet de leurs propres craintes ont de meilleurs contacts avec eux, ce qui facilite les interventions (O’Donnell et Waldo, 2005).

Un autre aspect qui amène des difficultés dans le travail auprès des pères est la répartition inégale et culturellement inhabituelle du pouvoir entre, d'une part, une intervenante qui agit au nom de la loi, et d'autre part, un homme en position de vulnérabilité (Baum, 2015a). La socialisation différenciée peut aussi être responsable du fait que les hommes ont peur d'être jugés incompétents en tant que parent puisqu'ils sont moins sensibilisés aux soins aux enfants que les mères (Maxwell et al. 2012a).

Baum (2015a) et Scourfield (2014) ont enfin mis en lumière la nécessité pour les intervenants d'être conscients de la façon dont leurs expériences avec leurs propres pères, et avec les hommes en général, peuvent affecter leur perception du rôle des pères et leurs interventions avec les hommes. Leurs recherches démontrent que les intervenants eux-mêmes peuvent représenter des obstacles à l'engagement des pères dans les services. D'ailleurs, les groupes de parole pour les intervenants, créés pour qu'ils puissent exprimer les émotions que le travail avec les pères suscite et analyser leur relation avec leur propre père et les hommes dans leur vie en général, se sont avérés efficaces pour améliorer le 
travail des intervenants face à l'engagement des pères dans les services et diminuer les préjugés envers eux (Scourfield, 2014).

\section{Facilitateurs au développement des liens entre les intervenants et les pères}

Un des éléments identifié comme facilitateur est la conviction que les intervenants ont de l'utilité de la participation des pères dans les interventions. Aussi, quand les pères sentent que l'intervenant est à leur écoute et les traite comme la mère, de façon égalitaire, ils sont plus enclins à s'investir (Devault et al, 2015b; Fletcher et St-George, 2010). De plus, la transparence, l'ouverture, la confiance, le travail d'équipe, le partage de la solution au problème contribuent à renforcer une relation positive (Lévesque, Moreau, Panet-Raymond et Roy, 1985).

Plusieurs études (Bayley, Wallace et Choudhry, 2009; Devault et al, 2015b) indiquent que les intervenants qui réussissent le mieux à entretenir une relation de confiance avec les pères, sont ceux qui font preuve d'ouverture, de non-jugement, d'empathie et de respect. Dans ce contexte, le père sent qu'il est écouté, respecté et qu'il a de la crédibilité aux yeux de l'intervenant. Si ce dernier est transparent et consulte le père pour la mise en place de l'intervention, le père s'engagera plus facilement dans les interventions proposées.

La collaboration semble également facilitée lorsque le père indique qu'il prend à cœur le bien-être de son enfant et qu'il est capable de se mobiliser pour les interventions. Cette mobilisation permet de dépasser les préjugés négatifs des intervenants à leur encontre qui les incite à une attitude plus ouverte à leur égard. Ainsi, la diminution des attitudes défensives de part et d'autre fait place au développement d'une relation davantage fertile à l'élaboration d'un terrain commun qui minimise en quelque sorte les effets délétères de la socialisation masculine (par ex. : protection de l'autonomie aux dépends de toute demande d'aide) et les préjugés potentiels des intervenants à l'encontre des pères (par ex. : incapacité des pères à se mobiliser).

D'autres études confirment que lorsque l'intervenant s'intéresse au père comme une personne, et non pas seulement pour les risques qu'il peut représenter pour la sécurité de l'enfant, il se montre plus ouvert à collaborer. Ainsi, prendre le temps de questionner le père sur son histoire de vie, sur ses valeurs, sur l'importance des enfants dans sa vie et sur ce qu'il est prêt à faire pour eux peut favoriser l'établissement d'un lien de confiance qui facilite les interventions (Baum, 2015a).

De plus, essayer de gagner la confiance des pères plutôt que de tenter d'exercer un pouvoir sur eux s'est révélé judicieux (Pleau, 2013). Par ailleurs, adopter une attitude de «non expert » permettrait aux 
intervenants d'être plus ouverts aux perceptions du père de sa propre situation. Être en mode «curiosité » et «exploration» favorise la réciprocité dans la relation parent-intervenant. D'autres auteurs, enfin, relèvent que les hommes souhaiteraient plus d'empathie de la part de l'intervenant (Scourfield, 2014). Ils aimeraient également pouvoir avoir l'opportunité de s'exprimer librement sur ce qu'ils vivent sans être immédiatement identifiés comme un homme violent (Campbell et al, 2015).

\section{Discussion}

Plusieurs facilitateurs de la collaboration pères-intervenants soulignés dans cette recension des écrits trouvent un écho dans la théorie de la reconnaissance (« recognition theory») décrite par Turney (2012). Cette théorie soutient que la reconnaissance, le respect et la réciprocité sont nécessaires à la compréhension de l'intervention en contexte d'autorité. Ainsi, c'est à travers l'expérience relationnelle de la reconnaissance de soi par l'autre que l'individu apprend à se (re)connaître. Les attitudes constructives que nous avons identifiées dans cette recension relèvent également du respect dont l'intervenant fait preuve à l'égard du père et qui contribue au fait que ce dernier se sente accepté comme partenaire de l'intervention. Dans ce contexte, respect et reconnaissance de la part des intervenants peuvent soutenir la parole du père, sa légitimité et sa mobilisation dans les interventions. La réciprocité, quant à elle, sous-tend qu'il est nécessaire que les échanges entre les pères et les intervenants puissent donner lieu au recul des préjugés entretenus de part et d'autre.

Pour ce qui est des pistes d'intervention, le rapport de Roy et ses collègues (2014) suggère de mettre en place des mesures de déconstruction des règles de la masculinité traditionnelle auprès des intervenants de manière à ce qu'ils comprennent la distance entre les attentes associées à la masculinité hégémonique et celles associées aux contacts avec les services sociaux. Par exemple, les professionnelles sont plus familières avec les manifestations de détresse féminine mais doivent être conscientes que les hommes peuvent vivre aussi leur détresse, même si c'est différemment. Les recherches démontrent que lorsque les intervenantes travaillent avec les hommes, elles ont de la difficulté à fournir le même degré de soutien émotionnel que celui qu'elles donneraient aux femmes (Baum, 2015b), voire elles minimisent ou ignorent la détresse des hommes. Notamment, certains hommes peuvent avoir tendance à «agir » leurs émotions par manque de symbolisation plutôt que de les exprimer verbalement. Ces «passages à l'acte» ne devraient pas d'emblée être perçus comme des indices de difficultés plus sévères. De façon générale, les hommes auraient tendance à vivre leurs émotions plus tardivement et à les gérer de façon plus indirecte par des moyens qui leur sont propres (consommation de substances ou travail acharné). Plusieurs ont aussi tendance à hausser le ton de la voix pour exprimer des émotions douloureuses (Baum, 2015a). Enfin, ils peuvent se montrer plus méfiants pour dévoiler leur intimité. Ces attitudes, avec lesquelles les intervenants sont moins familiers, ne peuvent qu'envenimer la relation (Scourfield, 2014). 
Roy et ses collaborateurs (2014) confirment l'importance d'adopter, en particulier avec les hommes, des approches centrées sur les forces et l'empowerment, basées sur le non-jugement et le respect (Deslauriers, 2008). Les hommes rechercheraient une forme d'échanges avec les intervenants, dans lesquels ils pourraient avoir la possibilité de prendre la parole et surtout qu'elle soit prise en considération. Ces auteurs utilisent le terme «horizontalité des services », par comparaison à un modèle vertical de services, pour décrire un fonctionnement institutionnel qui respecterait le besoin des hommes de maintenir une certaine autonomie et,un certain «contrôle » de la situation. Ce type de rapport entre institution et père mettrait les compétences des pères au service de l'intervention (Pouliot et Saint-Jacques, 2005). .

Enfin, à l'instar de Shafer et Wendt (2015), il est pertinent d'apporter quelques nuances au sujet de la masculinité hégémonique. D'une part, certaines attitudes traditionnellement masculines ne devraient pas être automatiquement associées à des barrières à la demande d'aide. Par exemple, le fait d'être orienté vers les solutions, de vouloir régler des problèmes, d'être axé sur la tâche représente des stratégies qui peuvent se révéler bénéfiques en intervention. D'autre part, il serait inexact de prendre pour acquis que tous les hommes évoluent sous l'égide de la masculinité hégémonique. Ainsi, d'autres facteurs doivent être pris en compte pour comprendre les enjeux vécus par les hommes comme par exemple les situations de pauvreté ou de précarité (Zaouche-Gaudron, Jayr et Kettani, 2005). Enfin, d'autres facteurs ont une influence sur le rapport des hommes à leur santé, notamment le revenu, le niveau de scolarité, le type d'emploi, l'âge, les liens sociaux et l'origine ethnique (Zaouche Gaudron, 2016). Il y a fort à parier que ces facteurs influencent également le rapport des hommes aux institutions et qu'il convient de mieux les apprécier afin que les interventions pour « faire avec » eux soient les plus efficaces possibles pour l'ensemble des membres de la famille.

\section{Conclusion}

Soutenir les pères dans un contexte dans lequel l'enfant est la cible de l'intervention est une pratique relativement récente, notamment en raison de leur absence - ou de leur évincement - des dispositifs lorsqu'il est question du bien-être des enfants. Cette recension d'écrits a voulu combler un certain vide quant à la documentation entourant les facteurs qui influencent l'établissement d'une relation entre un intervenant et un père lorsque l'enfant de ce dernier est suivi par les services de protection de l'enfance. Un certain nombre d'indications quant aux stratégies favorisant l'harmonie de ces liens ont été fournies par cette recherche. Ce faisant, cet article n'a pas eu pour visée de développer une perspective plus clinique qui aurait pu rendre compte des mécanismes de transfert-contre transfert à l'œuvre dans le champ interventionnel. Pour autant, les résultats des écrits recensés que nous avons évoqués pourraient être repris afin d'examiner, ce qui, dans le champ de la clinique, fait obstable à leur prise en charge dans le domaine de la protection de l'enfance. 
Pour se préparer au travail à réaliser avec des pères, il serait pertinent que les intervenants prennent le temps d'examiner leurs perceptions et croyances à l'égard des pères et leur rapport avec les enfants de manière à clarifier là où ils se situent, voir ce qui leur appartient et prendre une distance par rapport à certains préjugés défavorables de manière à proposer un autre regard sur les pères avec lesquels ils travailleront. Il serait aussi utile de réfléchir à l'insécurité que peuvent vivre certains pères. Malgré les contraintes associées à l'intervention en contexte d'autorité, ce genre de pratique dans laquelle l'intervenant cherche à connaître la personne qui se trouve devant lui peut être d'un grand secours pour développer un lien de confiance et pour obtenir la collaboration du père dans les démarches à entreprendre.

Sur le plan des interventions elles-mêmes, cette recension a indiqué qu'un mode d'intervention impersonnel, procédural ou basé sur la confrontation où il y a peu de place pour l'écoute, l'empathie et la participation des pères aux décisions constituent des stratégies d'intervention qui ont davantage de risque de mener à l'échec de la relation. Par opposition, la transparence de l'intervenant quant aux démarches à entreprendre, à ce qu'il sait, à ses obligations professionnelles compte parmi les qualités les plus importantes que les intervenants devraient développer.

Enfin, cette contribution laisse supposer que les services de protection doivent s'ouvrir aux pères, faire preuve d'une écoute attentive à leur réalité et à leur désir d'être pris en considération. Elle avance également que le désir de collaboration des pères aux mécanismes de l'institution est facilité par cette ouverture institutionnelle suivant un processus de réciprocité. Ainsi, le père qui se sent respecté et entendu au sein de l'institution sera plus à l'aise d'exprimer son désir de contribuer au processus d'intervention, et la perception d'une telle ouverture contribuera à diminuer les préjugés défavorables envers les pères. La réciprocité et la circularité établies contribueront à ce que les pères soient considérés comme un parent à part entière dans les services de protection de l'enfance.

\section{Bibliographie}

BAUM, N. 2015a. «Gender-sensitive intervention to improve work with fathers in child welfare services », Child and family Social Work, septembre 2015, 1-9.

BAUM, N. 2015b. «The Unheard Gender: The Neglect of Men as Social Work Clients », British Journal of Social Work, septembre 2015,1-9.

BAYLEY, J., WALLACE, L.M. \& CHOUDHRY, K. 2009. «Fathers and parenting programmes: barriers and best practice », Community Practitioner, 82, 4, 28-31.

BELLAMY, J.L. 2009. «A national study of male involvement among families in contact with the child welfare system », Child Maltreatment, 14, 255-262.

BROWN, L., STREGA, S., CALLAHAN, M., DOMINELLI, L. \& WALMSLEY, C. 2009. « Fathering within Child Welfare », Canada's Children, Centre of Excellence for Child Welfare, University of Toronto, 15, 3 .

CAMPBELL, C.A., HOWARD, D., RAYFORD, B.S. \& GORDON, D.M. 2015. « Fathers matter: Involving and engaging fathers in the child welfare system process », Children and Youth Services Review, 53, 84-91. 
DEMONTIGNY, F., DEVAULT, A. \& FLEURANT, A. 2009. « L'enseignement des enjeux de la paternité dans les universités canadiennes », Reflets, 16, 102-119.

- DESLAURIERS, J.-M. 2008. « Paternités exclues et la formation en travail social », Les Politiques sociales, 1-2, 83-98.

DEVAult, A., FORGET, G. \& DUBEAU, D. 2015a. Fathering. Promoting positive father involvement, Toronto, University of Toronto Press.

DEVAUlT, A., HUARD-FlEURY, M.C., MONETTE DREVILlON, M.F., LACHARITE, C., DEMONTIGNY, F. \& DUBEAU, D. 2015b. «"Can you hear me, Major Tom?” Les liens entre les pères et les intervenants dont les enfants sont sous les soins des services de protection de l'enfance », dans C. Lacharité et C. Sellenet (sous la direction de), La protection de l'enfance, Montréal, Presses de l’Université du Québec, 250-266.

DOMINELli, L., STREGA, S., WALMSLEY, C., CALLAHAN, M. \& BROWN, L. 2011. "'Here's my Story' Fathers of 'Looked After' Children Recount their Experiences in the Canadian Child Welfare System ", British Journal of Social Work, 41, 2, 351-367.

DUBEAU, D., DEVAUlT, A., FORGET, G. \& BIZOT, D. 2009. La paternité au XXIème siècle, Québec, Presses de l'Université Laval.

EWART-BOYLE, S., MANKTELOW, R. \& MCCOLGAN, M. 2013. « Social work and the shadow father; lesson for engaging fathers in Northern Ireland », Child and Family Social Work, 1-10.

FLETCHER, R.J. \& ST-GEORGE, J.M. 2010. « Practitioners' understanding of father engagement in the context of family dispute resolution », Journal of Family Studies, 16, 2, 101-115.

FORRESTER, D. \& HARWIN, J. 2008. « Parental substance misuse and child welfare: outcomes for children two years after referral », British Journal of Social Work, 38, 1518-1535.

GUAY, J. 2010. «Les familles récalcitrantes en protection de la jeunesse », Santé mentale au Québec, 35, 47-59.

HOWE, D. 2010. « The safety of children and the parent-worker relationship in cases of child abuse and neglect », Child Abuse Review, 19, 5, 330-341.

LACHARITE, C. \& GAGNIER, J.P. 2015. «Agir auprès des pères en situation de vulnérabilité : une invitation au dialogue et à la réflexion », Cahiers critiques de thérapie familiale et de pratiques de réseaux, 54, 81105.

LEVESQUE, J., MOREAU, M., PANET-RAYMOND, J. \& ROY, L. 1985. « Le rôle de l'intervenant(e): la relation d'aide dans l'approche structurelle, un rapport dialogique. Méthodologie de l'intervention », dans Recueil de textes, École de service social, Université de Montréal, 128-130.

MAU CHAN, T. 2012. «The key challenges of working with Chinese men: social worker accounts », Journal of Social Work Practice, 26, 2, 215-232

MAXWELL, N., SCOURFIELD, J., FEATHERSTONE, B., HOLLAND, S. \& TOLMAN, R. 2012a. « Engaging fathers in child welfare services: a narrative review of recent research evidence ", Child and Family Social Work, 17,160-169.

MAXWELL, N., SCOURFIELD, J., HOLLAND, S., FEATHERSTONE, B. \& LEE, J. 2012b. « The Benefits and Challenges of Training Child Protection Social worker in father Engagement », Child Abuse Review, $21,4,299-310$

O'DONNELL, J.M.J. \& WALDO, E. JR. 2005. « Fathers in child welfare: caseworkers' perspectives », Child welfare: journal of policy, practice, and program, 3-4, 84, 387-414. 
O'HAGAN, K.1997. «The problem of engaging men in child protection », British Journal of Social Work, 2, $25-42$.

PLEAU, A. 2013. Les effets de la judiciarisation sur l'implication parentale en protection de la jeunesse : Perceptions des intervenants, Mémoire de maîtrise, Université Laval.

POULIOT, E. \& SAINT-JACQUES, M.C. 2005. «L'implication des pères dans l'intervention en protection de la jeunesse : Un discours et une pratique qui s’opposent », Enfances, Familles, Générations, 3, 146-162.

ROY, J., TREMBlay, G., GUILMETTE, D., BIZOT, D., DUPERE, S. \& HOULE, J. 2014. Perceptions des hommes québécois de leurs besoins psychosociaux et de santé ainsi que leur rapport aux services. Métasynthèse, Québec, Gouvernement du Québec.

SALEH, M. F. 2013. « Child welfare professionals' experiences in engaging fathers in services », Child and Adolescent Social Work Journal, 30, 2, 119-137.

SCOURFIELD, J. 2003. Gender and Child Protection, Houndmills, Basingstoke, Palgrave MacMillan.

SHAFER, K. \& WENDT, D. 2015. «Men's Mental Health: A Call to Social Workers », Social Work, 60, 2, 105112.

TREMBLAY, G. \& DERY, F. 2011. «La santé des hommes au Québec », dans J.M. Deslauriers, G. Tremblay, S. Genest Dufault, D. Blanchette et J.-Y. Desgagnés (sous la direction de), Regards sur les hommes et les masculinités : comprendre et intervenir, Québec, Presses de l’Université Laval, 305-330.

TURCOTTE, G. 2014. « Coup d'œil sur l'engagement paternel. Faire place aux pères dans l'intervention en protection de la jeunesse : enjeux, défis et pistes d'action », Source : < http://observatoiremaltraitance.ca/Pages/Coup_d\%27oeil_sur_1\%27engagement_paternel.aspx/>

TURNEY, D. 2012. «A relationship-based approach to engaging involuntary clients: The contribution of recognition theory », Child et Family Social Work, 17, 2, 149-159.

WELZER-LANG, D. \& ZAOUCHE GAUDRON, C. 2011. Masculinités : état des lieux, Toulouse, Eres.

ZANONI, L., WARBURTON, W., BUSSEY, K. \& MCMAUGH, A. 2013. « Fathers as 'core business' in child welfare practice and research: An interdisciplinary review », Children and Youth Services Review, 35 , $1055-1070$

ZAOUCHE-GAUDRON, C., JAYR, P. \& KETTANI, M. 2005. «Expérience paternelle en situation de précarité économique », Sciences de l'Homme et Sociétés, 81, 42-44.

ZAOUCHE-GAUDRON, C. (ED.) (2016-A PARAITRE). Enfants de la précarité, Toulouse : Editions Eres. 